\title{
Differences in Heartbeat Modulation between Excited and Relaxed Kawaii Feelings during Photograph Observation
}

\author{
Miyuki YANAGI*, Yoshiyuki YAMARIKU**, Tomomi TAKASHINA**, \\ Yoshikazu HIRAYAMA**, Ryota HORIE* and Michiko OHKURA* \\ * Shibaura Institute of Technology, 3-7-5 Toyosu, Koto-ku, Tokyo 135-8548, Japan \\ ** Nikon Corporation, 1-12-1 Yurakucho, Chiyoda-ku, Tokyo 100-8331, Japan
}

\begin{abstract}
Kawaii describes Japanese culture and is used all over the world to express emotional feeling. This study hypothesized that kawaii feelings can be classified as excited and relaxed responses when watching photographs. Heart rate measurements were performed for subjects viewing exciting kawaii, relaxing kawaii, and uninteresting photographs. Statistical tests were conducted to relate heartbeat modulations and photograph conditions. Exciting kawaii photographs enhanced heart rates, while their relaxing counterparts did not increase heart rates to the same levels, validating the initial hypothesis.
\end{abstract}

Keywords: Kawaii feeling, Heart rate, Photographs

\section{INTRODUCTION}

Kawaii has become a descriptive word for Japanese culture and is used all over the world to express emotional feelings. Translated into cute, loveable, and adorable, kawaii has been given multiple definitions in recent years depending on a person's taste and the given situation [1]. Various aspects of kawaii have been discussed in several fields such as psychology, sociology, and Kansei Engineering. Systematic Kansei Engineering studies have addressed kawaii objects and attributes and revealed that physiological responses evoked by the kawaii feeling are measurable in heartbeat and electroencephalogram [2]. These scientific results have recently led to industrial products [3], demonstrating their significance.

A person experiences kawaii feelings when observing kawaii objects or attributes in everyday sceneries. Specifically, watching photographs elicits subjective, intuitive, and strong preferences depending on personal emotional feelings, suggesting that investigation of kawaii feeling that occur while watching photographs provides valuable insights. Therefore, kawaii-related physiological changes while performing this task were measured. In a previous study, we detected heartbeat modulation when subjects experienced kawaii feeling when watching photographs $[4,5]$. A pronounced modulation was observed when the subjects themselves selected kawaii photos that act as stimuli. Moreover, physiological brain responses were measured in event-related potentials when subjects experienced kawaii feeling when watching photographs [6,7].
In the fields of physiology and bioengineering, a number of recent studies demonstrated that affective responses measured in biosignals can be mapped and classified on a tow dimensional space of the Russell's circumplex model $[8,9]$. Although various types of kawaii feeling have been known [1], physiological evidence for classification of kawaii feeling has not been well studied. Thus, the goal of this study was to show the physiological evidence for classification of kawaii feeling while watching photographs. The classification by biosignals is necessary and significant to realize precise evaluation of kawaii feeling toward development of industrial products [3] and interfaces as in an emotion driven digital camera [7]. Excited and relaxed feelings are typical feelings obtained while watching kawaii photographs. The excited and relaxed feelings are represented in opposite positions along the arousal axis in the Russell's circumplex model and cause increase and decrease in heart rate respectively [8]. Thus we hypothesized that the kawaii feeling evoked while watching photographs could be classified into two types [10]. The first type, called "excited kawaii" in this paper, corresponds to a feeling of cuteness accompanied by excitation. The second type, or "relaxed kawaii," is a feeling of cuteness accompanied by relaxation. We further hypothesized that the two types of feelings can be measured as changes of heart rate. To test this hypothesis, in this study, heartbeat modulations were measured when subjects experienced both types of kawaii feeling while watching photographs and potential differences in these heartbeat modulations were examined with respect to both types. 


\section{MATERIAL AND METHODS}

\subsection{Subjects}

Seven healthy subjects in their 20s participated in the experiment. All subjects gave informed consent prior to participation. The experimental procedure was approved by the ethics committee of Shibaura Institute of Technology.

\subsection{Stimuli}

Each subject subjectively selected (1) an exciting kawaii photograph, which evoked excited kawaii feeling, (2) a relaxing kawaii photograph, which evoked relaxed kawaii feeling, and (3) an uninteresting photograph, which evoked no specific feeling. Exciting kawaii photographs were expected to make the subjects' hearts palpitate and in turn enhance their heart rates. Relaxing kawaii photographs were assumed to make the subjects unwind, resulting in lower heart rate increase compared with their exciting kawaii counterparts. Uninteresting photographs were not expected to cause any heartbeat modulation and acted as control stimuli. Heartbeat modulation differences according to the types of photographs would physiologically verify the hypothesis that the kawaii feeling resulting from watching photographs can be categorized as excited and relaxed.

Subjects were instructed to select photographs that induced the desired feelings in a stable and permanent manner from the Internet. Typical exciting kawaii photographs showed animals and portraits across the subjects while their relaxing kawaii counterparts usually displayed animals. Uninteresting photographs generally presented tools, furniture, buildings, or sceneries. Interestingly, one subject selected an animal picture as an exciting kawaii photograph that was chosen as a relaxing kawaii one by another subject. This suggested that the kawaii classification strongly depended on subjective feelings. Selected photographs were converted to monochrome images and edited to fit a 192 pixel-high by 256 pixel-wide frame.

\subsection{Heart rate measurements}

Visual stimuli were displayed on a laptop computer screen placed in front of the subjects (Figure 1). During each trial, subjects quietly faced a black screen for $30 \mathrm{~s}$ (rest period) before watching a picture for $30 \mathrm{~s}$ (task period). This trial was repeated five times for a total duration of $300 \mathrm{~s}$ while an electrocardiogram (ECG) was measured by radiofrequency ECG (Wireless Vital Sign Sensor, Micro Medical Device, Inc.). Heart rates were calculated from $\mathrm{R}-\mathrm{R}$ intervals in individual ECGs.

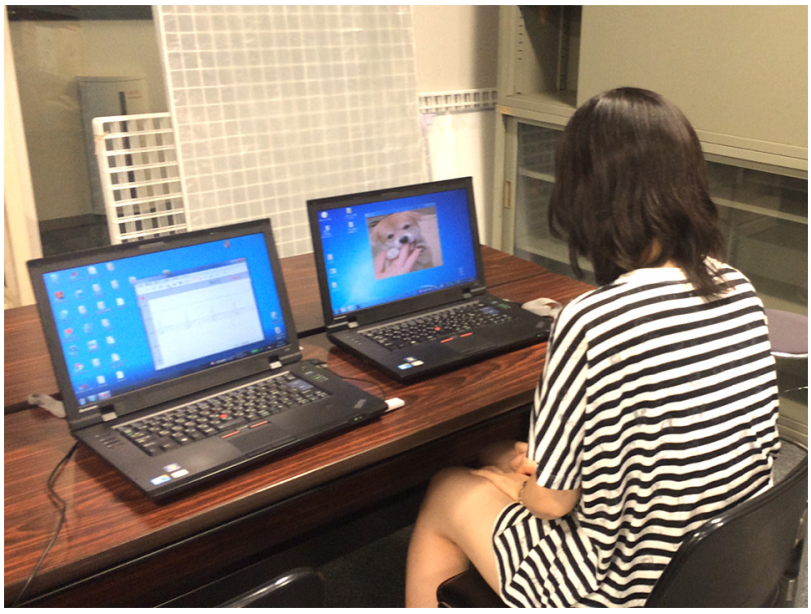

Figure 1: A snapshot of conducting the experiment

The period taken to present each picture was longer that than in previous experiments [2] in order to provide sufficient time to significantly change heart rates. The time interval between photograph selection and ECG measurement was limited to $10-15 \mathrm{~min}$ to ensure that the subject's impressions of the photos remained unchanged.

\section{RESULTS}

\subsection{ECG waveforms}

A typical ECG waveform (Figure 2) shows the amplitude $[\mathrm{mV}]$ as a function of time [s] within a trial. This waveform was extracted from a trial using an exciting kawaii photograph. Figures 3-5 show typical heart rates obtained during each trial for a subject using exciting kawaii, relaxing kawaii, and uninteresting photographs, respectively. In the Figures 3-5, numbers in legends are corresponding to the trials. Horizontal axes indicate the rest and task periods while vertical axes represent heart rates in a trial $[\mathrm{bpm}]$. Heart rate distributions are shown for each trial.

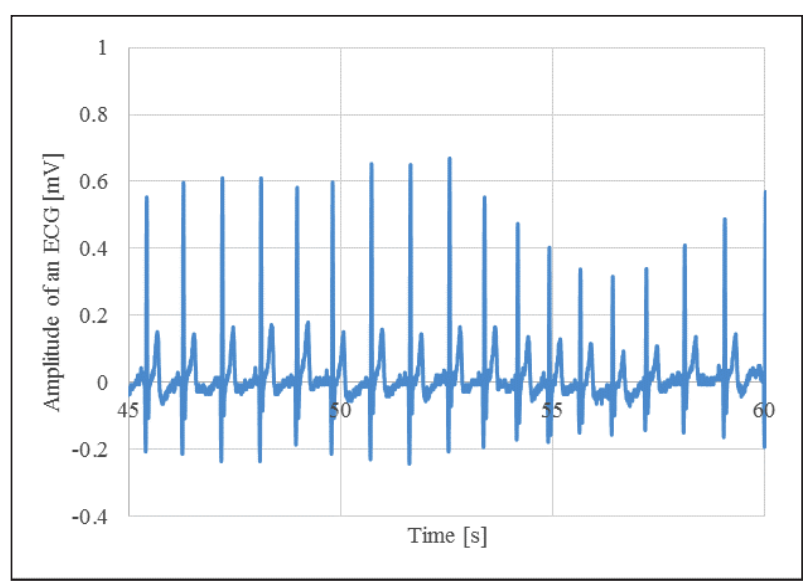

Figure 2: A typical waveform of the heartbeat 


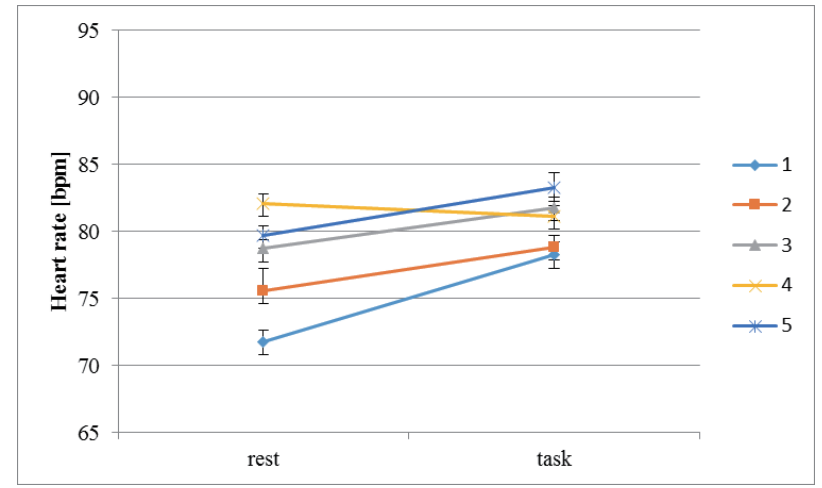

Figure 3: Typical heart rates for each trial using an exciting kawaii photograph

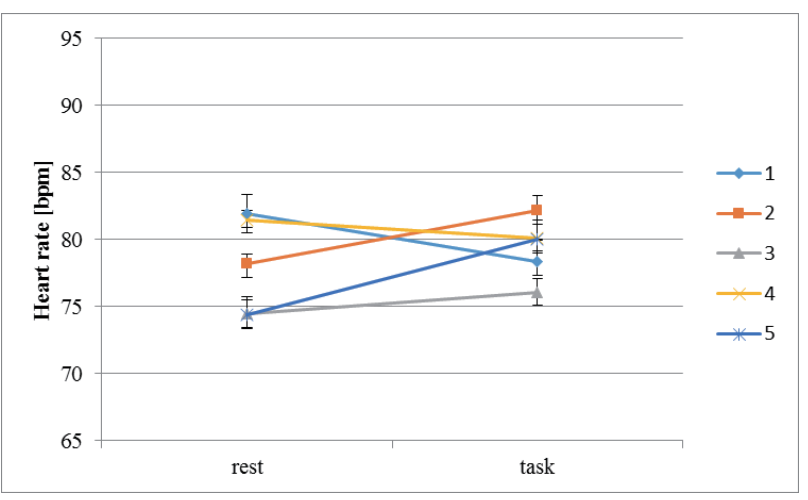

Figure 4: Typical heart rates for each trial using a relaxing kawaii photograph

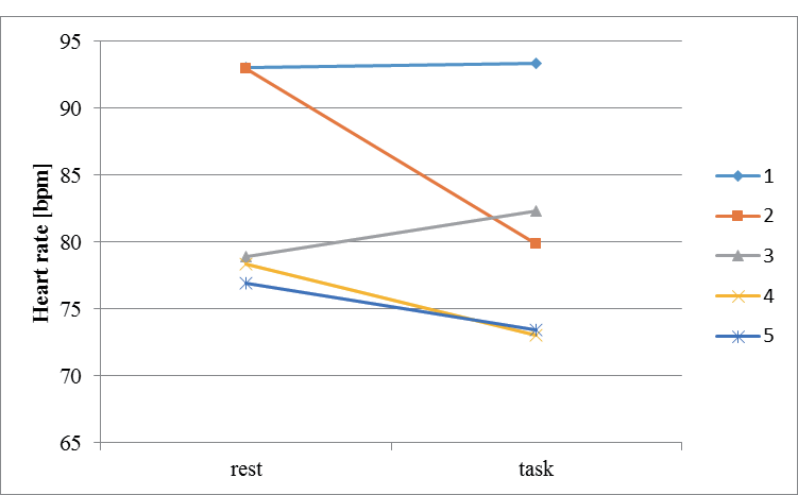

Figure 5: Typical heart rates for each trial using an uninteresting photograph

\subsection{Average heart rate difference}

For each trial, a heartbeat modulation evoked by a photograph was defined as a change in heart rate average during the second half of the task period (15 s), which was assumed as a baseline, relative to the heart rate average during the first half of the task period (15 s). This change was called heart rate difference. For each subject, five heart rate differences were obtained and averaged for the five trials conducted using individual pictures. These averages were defined as average heart rate differences (Table 1).
Table 1: Average heart rate differences for each type of photograph and individual subject

\begin{tabular}{|c|c|c|c|}
\hline Subjects & $\begin{array}{c}\text { excitedly } \\
\text { kawaii photo }\end{array}$ & $\begin{array}{c}\text { relaxingly } \\
\text { kawaii photo }\end{array}$ & $\begin{array}{c}\text { uninterenting } \\
\text { photo }\end{array}$ \\
\hline $\mathrm{A}$ & 3.063 & 1.266 & -3.623 \\
\hline $\mathrm{B}$ & 2.164 & 3.620 & -0.864 \\
\hline $\mathrm{C}$ & -0.960 & -1.919 & -2.700 \\
\hline $\mathrm{D}$ & 0.713 & 0.339 & 1.619 \\
\hline $\mathrm{E}$ & 1.695 & 1.612 & 0.555 \\
\hline $\mathrm{F}$ & 4.003 & -0.711 & -0.764 \\
\hline $\mathrm{G}$ & 2.155 & 1.261 & -1.580 \\
\hline \hline Average & 1.833 & 0.781 & -1.051 \\
\hline$p$-value & 0.024 & 0.289 & 0.173 \\
\hline
\end{tabular}

\subsection{Statistical tests}

Statistical tests were conducted using average heart rate differences (Table 1). To determine whether a type of photograph induced a heartbeat modulation, a t-test was performed for these average differences according to the stimulus. Exciting kawaii photographs produced a significant difference $(p<0.05)$, whereas relaxing kawaii and uninteresting photographs induced little changes.

ANOVA was conducted to evaluate whether heartbeat modulation depended on the type of photograph presented. A significant difference was observed between the three stimuli $(p<0.05)$. Table 2 shows post-hoc multiple comparison results of the one-way ANOVA using the Bonferroni correction. A significant difference was obtained between exciting kawaii and uninteresting photographs $(p<0.05)$. No significant differences were detected between exciting and relaxing kawaii photographs or between relaxing kawaii and uninteresting photographs. Figure 6 shows one-way ANOVA and post-hoc multiple comparison results. Average heart rate differences [bpm] are displayed for individual exciting kawaii, relaxing kawaii, and uninteresting photographs. Average heart rate difference distributions are also presented for the seven subjects.

Table 2: Post-hoc multiple comparison results of the one-way ANOVA

\begin{tabular}{|c|c|}
\hline & $p$-value \\
\hline excitedly kawaii photo and relaxingly kawaii photo & 0.811 \\
\hline excitedly kawaii photo and uninteresting photo & 0.018 \\
\hline relaxingly kawaii photo and uninteresting photo & 0.189 \\
\hline
\end{tabular}




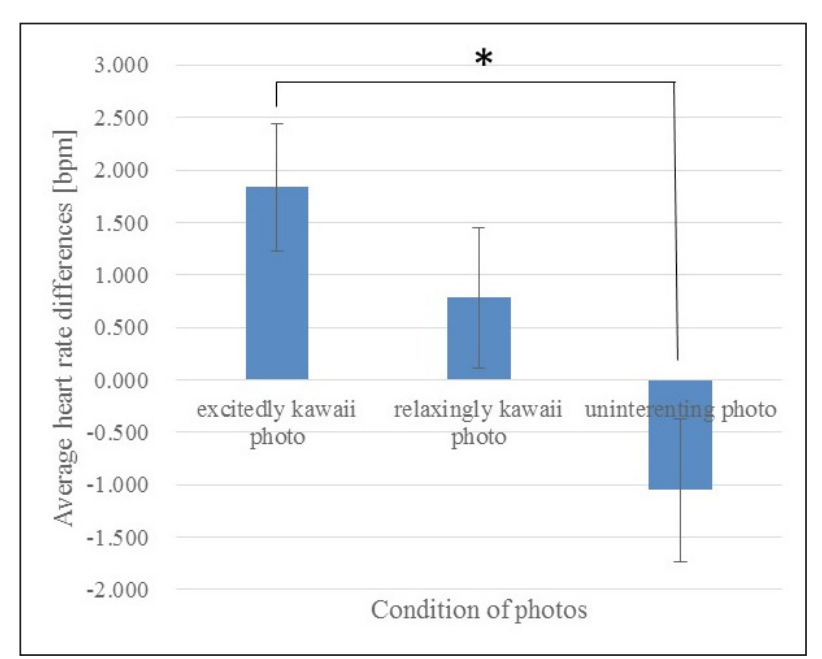

Figure 6: A typical waveform of the heartbeat

\section{DISCUSSION}

A significant t-test result was observed only for exciting kawaii photographs, indicating that these photographs increased heart rates. Moreover, significant differences were detected between exciting kawaii and uninteresting photographs, whereas relaxing kawaii and uninteresting photographs produced little changes. These results validate the hypothesis that the kawaii feeling evoked while watching photographs can be classified as excited and relaxed kawaii feelings. Exciting kawaii photographs produced a heartbeat modulation, unlike their relaxing kawaii counterparts.

The heart rate increase induced by the exciting kawaii photographs was consistent with previous observations for subjects watching cute pictures [4]. Here, relaxing kawaii photographs did not boost heart rates to the same extent as their exciting kawaii counterparts, showing that the relaxing effects of healing photographs may suppress the increase in heart rate [11]. Furthermore, we speculated that excited and relaxed kawaii feelings may correspond to two opposite directions on the arousal axis of Russell's circumplex model of emotion as we led our hypothesis.

\section{CONCLUSION}

This study verified the hypothesis that the kawaii feelings evoked while watching photographs can be classified as excited and relaxed kawaii feelings. Heartbeat modulations occurring in subjects experiencing these feelings while watching photographs were measured. Exciting kawaii photographs significantly enhanced heart rates, whereas their relaxing kawaii counterparts induced little heart rate increases, validating our hypothesis. Overall, the heartbeat responses resulting from watching kawaii photographs exhibit different modulations depending on the two types of kawaii feeling, although only one word is used in our daily lives.

\section{REFERENCES}

1. Inuhiko Yomota; "Kawaii" Ron, Chikuma Shobo, 2006 (in Japanese).

2. Michiko Ohkura, Sayaka Goto, Asami Higo and Tetsuro Aoto; Relation between Kawaii Feeling and Biological Signals, Journal of the Japan Society of Kansei Engineering, 10(2), pp.109-114, 2011.

3. Japan Society of Kansei Engineering; Kawaii Kansei design award. http://kawaii-award.org/

4. Miyuki Yanagi, Yousuke Yamasaki, Yoshiyuki Yamariku, Tomomi Takashina, Yoshikazu Hirayama, Ryota Horie and Michiko Ohkura; Relation between Kawaii Feeling and Heart rates in Watching Photos, 15th Japan Society of Kansei Engineering Conference and General Meeting, B12, Tokyo, 2013.

5. Ryota Horie, Miyuki Yanagi, Ryo Ikeda, Yousuke Yamasaki, Yoshiyuki Yamariku, Tomomi Takashina, Yoshikazu Hirayama and Michiko Ohkura; EventRelated Potentials Caused by Kawaii Feeling in Watching Photos Selected by Heart Beat Modulation, EMBC'14 Proc., TD18.19, Chicago, 2014.

6. Miyuki Yanagi, Yousuke Yamasaki, Yoshiyuki Yamariku, Tomomi Takashina, Yoshikazu Hirayama, Ryota Horie and Michiko Ohkura; Physiological Responses Caused by Kawaii Feeling in Watching Photos, Proc. AHFE2014, pp.839-847, 2014.

7. Tomomi Takashina, Miyuki Yanagi, Yoshiyuki Yamariku, Yoshikazu Hirayama, Ryota Horie and Michiko Ohkura; Toward Practical Implementation of Emotion Driven Digital Camera Using EEG, Proc. AH2014, Article No.3, 2014.

8. Jonathan Posner, James A. Russell, and Bradley S. Peterson; The Circumplex Model of Affect: An Integrative Approach to Affective Neuroscience, Cognitive Development, and Psychopathology, Development and Psychopathology, 17(3), pp.715734, 2005.

9. Sander Koelstra, Christian Muhl, Mohammad Soleymani, Jong-Seok Lee, Ashkan Yazdani, Touradj Ebrahimi, Thierry Pun, Anton Nijholt, and Ioannis Patras; DEAP: A Database for Emotion Analysis; Using Physiological Signals, Affective Computing, IEEE Transactionson, 3(1), pp.18-31, 2011 
10. Miyuki Yanagi, Yoshiyuki Yamariku, Tomomi Takashina, Yoshikazu Hirayama, Ryota Horie and Michiko Ohkura; Relation between Kawaii Feeling and Heart Rates in Watching Photos the second report - Change in the heart rate by the kind of kawaii photos-, 16th Japan Society of Kansei Engineering Conference and General Meeting, E11, Tokyo, 2014.

11. Gayl Pollard and Roderick Ashton; Heart Rate decrease: A Comparison of Feedback Modalities and Biofeedback with Other Procedures, Biological Psychology, 14(3-4), pp245-257, 1982.

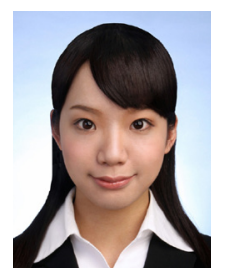

\section{Miyuki YANAGI}

Miyuki Yanagi received her B.S. (2013) in Communications Engineering and M.S. (2015) in Electrical Engineering and Computer Science from the Shibaura Institute of Technology, Japan. She currently works at Hewlett Packard Enterprise. Her research interests include biosignal measurements and fabrication workshop for understanding Kawaii feeling.

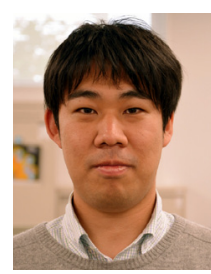

\section{Yoshiyuki YAMARIKU}

Yoshiyuki Yamariku is an associate researcher in MS Research Section, Research \& Development sector, Core Technology Division, Nikon Corporation, Japan. His research interests include image processing, computer vision, and computer graphics.

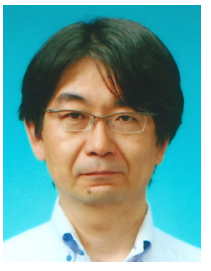

\section{Tomomi TAKASHINA}

Dr. Tomomi Takashina is a chief researcher at Nikon Corporation, Japan. His research and engineering work focuses on image processing and human computer interaction.

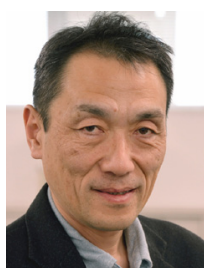

\section{Yoshikazu HIRAYAMA}

Yoshikazu Hirayama is a section manager in R\&D Administration Section, Research \& Development sector, Core Technology Division, Nikon Corporation, Japan. His research interests include applications of brain-computer interface.

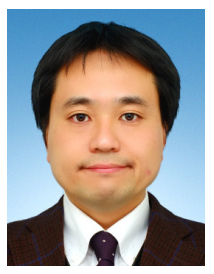

\section{Ryota HORIE}

Ryota Horie is an associate professor of Department of Communications Engineering, College of Engineering, the Shibaura Institute of Technology, Japan. His current research interests include brain-computer interface and their applications, natural user interface, and biosignal processing.

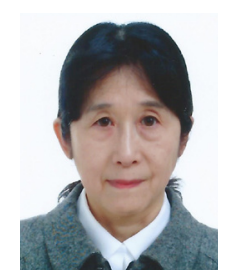

\section{Michiko OHKURA (Member)}

Michiko Ohkura received her B.S. (1976) and M.S. (1978) degrees in Mathematical Engineering and Ph. D. (1995) in Advances Interdisciplinary Studies from the University of Tokyo, Japan. She worked for some companies including Central Research Laboratory, Hitachi Ltd., and is currently a professor, Department of Information Science and Engineering, the Shibaura Institute of Technology, Japan. Her current research interests include human-friendly interactive systems and creation of Kansei values. 